\title{
CARACTERIZAÇÃO DE UMA MICROESTRUTURA POLICRISTALINA VIA DESCRITORES MICROESTRUTURAIS OBTIDOS POR SIMULAÇÃO *
}

\section{Resumo}

\author{
Guilherme Dias da Fonseca ${ }^{1}$ \\ Felipe da Silva Siqueira ${ }^{2}$ \\ André Luiz Moraes Alves ${ }^{1}$ \\ Marcos Felipe Braga da Costa ${ }^{3}$ \\ Beatriz Lemos Clemente ${ }^{2}$ \\ Júlia Mendes dos Santos ${ }^{2}$ \\ Eneias Pacheco Moreira Junior ${ }^{2}$ \\ Weslley Luiz da Silva Assis ${ }^{4}$ \\ Paulo Rangel Rios ${ }^{4}$
}

Com base nos modelos analíticos de Johnson e Mehl, Kolmogorov e Avrami (JMAK) e Cahn, no presente trabalho estudou-se via simulação computacional a cinética das transformações de fases no estado sólido. Tais transformações ocorreram numericamente por nucleação e crescimento nas interfaces de poliedros do tipo Ortotetracaidecaedros em um domínio discreto. $O$ intuito da matriz de Ortotetracaidecaedros é aproximar-se das características fenomenológicas de uma rede perfeita de grãos no espaço amostral. Realizaram-se simulações de nucleação com posições aleatórias em toda a matriz (bulk). Em um segundo estudo foram realizadas novas simulações de nucleação aleatoriamente, porém somente nas interfaces dos Ortotetracaidecaedros. A quantidade de núcleos iniciais para cada caso foi definida por processo de ponto de Poisson. A fração volumétrica, a área interfacial entre material transformado e não transformado, a contiguidade e o caminho microestrutural foram os resultados obtidos através das simulações. Tais resultados foram comparados com as equações analíticas. As nucleações com até mil núcleos corroboraram com JMAK e foram ratificados com o comportamento da contiguidade e do caminho microestrutural. Já as nucleações com valores acima de mil núcleos, mostram a presença de clusters, apresentando uma boa concordância com o modelo analítico de Cahn.

Palavras-chave: Simulação Computacional; Nucleação e Crescimento; Ortotetracaidecaedro; Parâmetros Microestruturais.

\section{CHARACTERIZATION OF A POLYCRISTALINE MICROSTRUCTURE VIA MICROSTRUCTURAL DESCRIPTORS OBTAINED BY SIMULATION}

\section{Abstract}

Based on analytical models of Johnson and Mehl, Kolmogorov and Avrami (JMAK) and Cahn, simulate yourself in the present work the kinetics of phase transformations in the continuous state by nucleation and growth in the interfaces of a polyhedral array of Orthotetracaidecahedrons. The purpose of the matrix is to approximate the phenomenological characteristics of a perfect grains network in the sample space. Simulations carried out random throughout the matrix and interfaces using as nuclei amount the values defined by Poisson. The volumetric fraction, the interfacial area between recrystallized and non-recrystallized material, the contiguity and the microstructural path were the results obtained through the simulations. These results were compared with the analytical equations. Nucleotides with up to a thousand nuclei corroborated with JMAK and were ratified with the behavior of contiguity and the microstructural path. Already the nucleations with values above a thousand 
nuclei, show the presence of clusters, presenting a good agreement with the analytical model of Cahn.

Keywords: Computational Simulation; Nucleation and Growth;

Ortotetracaidecahedro; Microstructural Parameters.

1 Engenharia Metalúrgica, Doutorando, estudante, Universidade Federal Fluminense, Núcleo de Modelamento Microestrutural, UFF-EEIMVR, Sala C87, Av. dos Trabalhadores, 420, CEP 27255125, Vila Santa Cecília, Volta Redonda, RJ, Brasil.

2 Engenharia Metalúrgica, Graduando, estudante, Universidade Federal Fluminense, Núcleo de Modelamento Microestrutural, UFF-EEIMVR, Sala C87, Av. dos Trabalhadores, 420, CEP 27255125, Vila Santa Cecília, Volta Redonda, RJ, Brasil.

3 Engenharia Metalúrgica, Mestre, estudante, Universidade Federal Fluminense, Núcleo de Modelamento Microestrutural, UFF-EEIMVR, Sala C87, Av. dos Trabalhadores, 420,CEP 27255125, Vila Santa Cecília, Volta Redonda, RJ, Brasil.

4 Engenharia Metalúrgica, Doutor, professor, Universidade Federal Fluminense, Núcleo de Modelamento Microestrutural, UFF-EEIMVR, Sala C87, Av. dos Trabalhadores, 420,CEP 27255125, Vila Santa Cecília, Volta Redonda, RJ, Brasil.. 


\section{INTRODUÇÃO}

As transformações de fase no estado sólido em materiais metálicos ocorrem em duas etapas. Na primeira, tem-se o surgimento de novas regiões, denominada de nucleação. Na segunda etapa, chamada de crescimento, essas novas regiões crescem a partir da matriz não transformada até que toda a matriz esteja completamente transformada. Uma das dificuldades nas simulações destas transformações é naturalmente o modelamento da nucleação.

O modelo analítico de Johnson e Mehl [1], Kolmogorov [2] e Avrami [3-5] (JMAK) é normalmente empregado em estudos da cinética de transformações de fases por nucleação e crescimento[6].

Geralmente, em casos reais, os locais preferenciais para a nucleação em materiais policristalinos são regiões de maior nível de energia armazenada tais como as arestas, vértices e faces dos grãos. Estas regiões são conhecidas como contornos de grãos ou sítios de nucleação. Desta forma, utilizou-se o método do cone causal para assim obter uma matriz em 3D de malhas quadráticas com as mesmas dimensões de um ortotetracaidecaedro, também conhecido como poliedro de kelvin, que pudessem se aproximar das características de uma rede policristalina.

Simularam-se os fenômenos de nucleação e crescimento de forma aleatória em toda a matriz e também nas interfaces dos grãos. A partir das simulações cinéticas foram caracterizados os seguintes parâmetros: fração volumétrica transformada $V_{V}$, área interfacial por unidade de volume em função do tempo $S_{v}$ e caminho microestrutural.

Os resultados foram comparados com os modelos analíticos de JMAK e de Cahn [7], sendo este último revisitado por Rios e Villa [8].

Através do estudo da contiguidade[9,10] foi possível caracterizar a distribuição espacial (dispersão) dos novos grãos incorporados em uma determinada transformação em termos da densidade de área dos contornos dos grãos. Este parâmetro foi determinante para à análise do caminho microestrutural [11,12], como sugeriu Vandermeer, [13].

\section{DESENVOLVIMENTO DOS MODELOS ANALÍTICOS EM 3D}

\subsection{A teoria de JMAK}

Os autores consideraram que o crescimento dos grãos da matriz $\alpha$ numa nova fase $\beta$, ocorrem sem a interferência (impigement) de uns contra os outros, ou seja, os grãos crescem ocupando toda a matriz e seu crescimento ocorre para dentro da região dos grãos vizinhos, denominado como volume estendido $V_{E}$. Porém, sabe-se que quando comparado com ao volume real, o $V_{E}$ possui maior volume por considerar o crescimento para além do grão.

Já nas transformações reais, os núcleos competem entre si para evoluírem até que toda à matriz esteja transformada. A partir das considerações matemáticas entre o volume estendido e o real, é obtida a fração volumétrica real ou fração recristalizada $V_{v}$. Para a nucleação distribuída de forma aleatória no espaço $V v(t)$ são obtidas as equações 1 e 2 .

$$
\begin{aligned}
& V_{V}=1-\exp \left(-V_{E}\right) \\
& V_{V}(t)=1-\exp \left(-k t^{n}\right)
\end{aligned}
$$


A equação 2 é comumente conhecida como equação generalizada de JMAK, sendo $V_{V}$ a fração volumétrica, $t$ o tempo da transformação, $k$ a constante de forma dos núcleos que crescem em função do tempo e $n$ representa a constante de Avrami.

Considerando a nucleação por saturação de sítios em que todos os núcleos são formados no instante inicial $(t=0)$. A equação 2 pode ser estendida da seguinte forma:

$$
V_{V}(t)=1-\exp \left(-\frac{4 \pi}{3} N_{V} G^{3} t^{3}\right)
$$

Sendo $N v$ o número de núcleos por unidade de volume e $G$ a velocidade de crescimento.

\subsection{Modelo de Cahn}

O modelo de Cahn trata do estudo da nucleação e crescimento de um policristal constituído de grãos equiaxiais pelo qual foi considerado que as faces, arestas e vértices são, respectivamente, planos aleatórios, linhas aleatórias e pontos uniformemente aleatórios no espaço.

Rios e Villa revisitaram o trabalho original e derivaram expressões para a cinética de transformação, onde a nucleação ocorre por saturação de sítios em planos paralelos obtendo assim a equação 4.

$$
V_{V}(t)=1-\exp \left(-2 S_{V}^{\text {planos }} G t \int_{0}^{1}\left\{1-e^{-\pi \lambda s G^{2} t^{2}\left[1-z^{2}\right]}\right\} d z\right)
$$

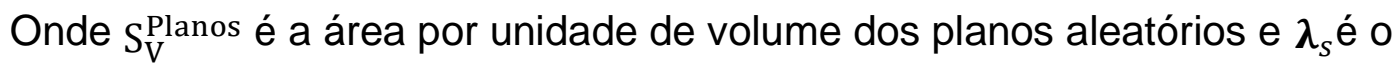
número de núcleos por unidade de área dos planos.

\section{MATERIAIS E MÉTODOS}

Utilizou-se no presente trabalho programação computacional em linguagem Fortran 2003, compilado pelo Microsoft Visual Studio2012®, com os seguintes recursos de hardware e software: computador com dois processadores físicos Intel Xeon E5-2650 v2® $2.60 \mathrm{GHz} 64$ bits, memória Ram 128 Gb e HD de 2 Tb em sistema operacional Windows Server 2008 R2®, e computador com processador Intel Core $17 \AA 3.40 \mathrm{GHz}$, memória Ram $32 \mathrm{~Gb}$ e HD de $2 \mathrm{~Tb}$ em sistema operacional Windows 8.1 Pro®. Para maior agilidade das simulações, empregou-se os recursos da paralelização em Open MP. Na geração e visualização das microestruturas, utilizou-se os programas Tecplot $360 \AA$ e o Wolfram Mathematica 11®.

A matriz considerada foi de $304 \times 304 \times 304$ células. As simulações de nucleação e crescimento nas interfaces do poliedro de Kelvin foram realizadas de forma aleatória, utilizando as variáveis definidas por Poisson. A nucleação ocorreu por saturação de sítios, com velocidade de crescimento constante e igual para todos os núcleos. O formato dos grãos foi considerado como esféricos.

\subsection{Cone Causal}

A regra de transição do cone causal em 3D se dá através de iterações ao longo do tempo. Para ocorrer a transformação de um ponto qualquer na matriz é necessário haver pelo menos um núcleo dentro da esfera de raio $\mathrm{R}$ que é centrada 
neste ponto qualquer. $\mathrm{O}$ raio $\mathrm{R}$ dessa esfera aumenta com o tempo e à medida que isto acontece, em cada interação de tempo, maior a possibilidade da existência de núcleos dentro da esfera.

Para cada interação o programa analisa todos os pontos da matriz para a verificação de quais serão transformados

$\mathrm{Na}$ figura 1 têm-se uma representação hipotética do modelamento do crescimento em 3D, onde o ponto $(1,1,1)$ é analisado. Os pontos da matriz são representados em vermelho e os núcleos em azul, porém a esfera de raio $\mathrm{R}$ está sendo representada por uma circunferência.

Em 1 (a) o tempo é $t=1$ e $R=1$ e não há nenhum núcleo transformado dentro da circunferência e por isso o ponto $(1,1,1)$ continua não transformado. Em 1 (b) e (c) o tempo é $t=2, t=3$ e $R=2, R=3$, respectivamente, e ainda não houve transformação. Em 1(d) o tempo é $t=4$ e $R=4$ e ocorre a transformação do ponto $(1,1,1)$ porque agora existe um núcleo transformado dentro da circunferência. Este processo ocorre ao mesmo tempo para todos os pontos da matriz em cada interação de tempo.

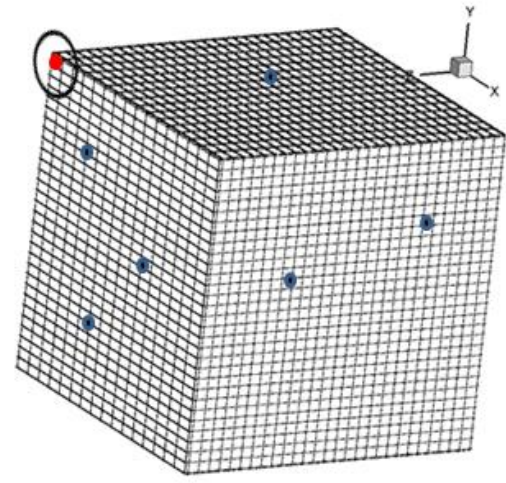

(a)

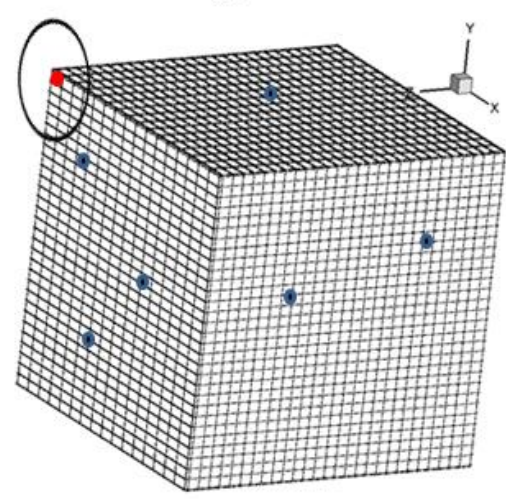

(c)

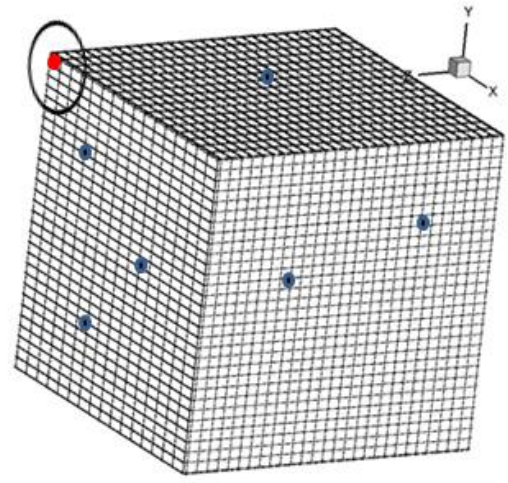

(b)

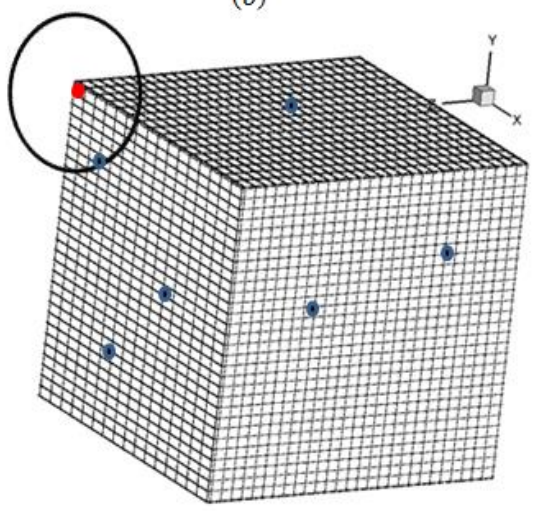

(d)

Figura 1 - Representação hipotética da teoria do cone causal entre os pontos da matriz - (a) Tempo $t_{1}$, (b) Tempo $t_{2}$, (c) Tempo $t_{3}$, (d) Tempo $t_{4}$, (interceptando um núcleo). Tem-se $t_{4}>t_{3}>t_{2}>t_{1}$.

\section{RESULTADOS E DISCUSSÃO}

Para a comparação entre os resultados das simulações aleatórias nas interfaces dos ortotetracaidecaedros com os resultados obtidos das simulações 
aleatórias na matriz, foram realizadas 50 simulações de nucleação e crescimento para cada caso.

Os resultados obtidos das simulações foram comparados com as equações (3) e (4), referente ao modelo analítico JMAK e de Cahn, respectivamente. As unidades de tempo e comprimento utilizadas no estudo são adimensionais. O tempo é discreto na simulação, partindo de $\mathrm{t}=0$ até a transformação total da matriz.

$\mathrm{Na}$ figura 2, tem-se a representação da microestrutura decorrente das transformações por nucleação e crescimento nas interfaces da malha de ortotetracaidedros contendo 8 núcleos. Os resultados foram obtidos em 3D, porém são apresentados em 2D.

Os grãos crescem com velocidade constante e seu formato inicial é aproximadamente esféricos.

$\mathrm{Na}$ figura $2 \mathrm{a}$, tem-se a nucleação no instante $\mathrm{t}=0$, porém, os núcleos estão fora do plano xy portanto não é possível visualizá-los. Em $2 b$ é possível observar os grãos com formato esférico, a aleatoriedade entre eles e a não ocorrência do impingement.

A partir de 2c, é possível observar que os núcleos começam a perder o formato esférico devido ao impingement. Assim, em $2 \mathrm{f}$, temos a matriz totalmente transformada.

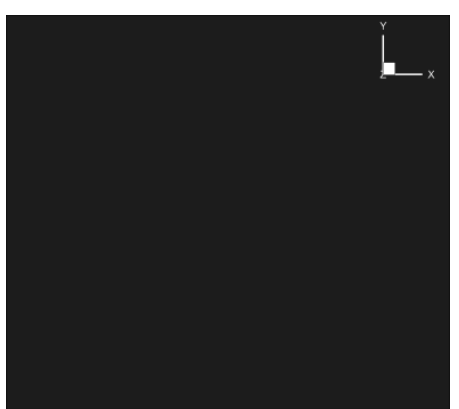

(a)

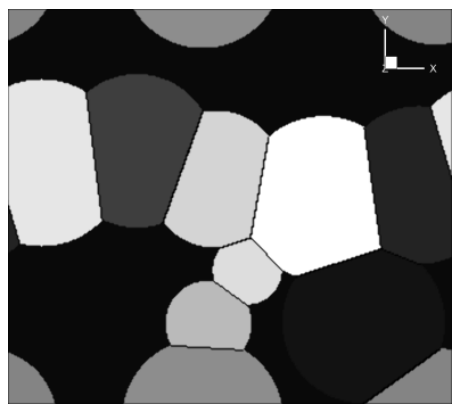

(d)

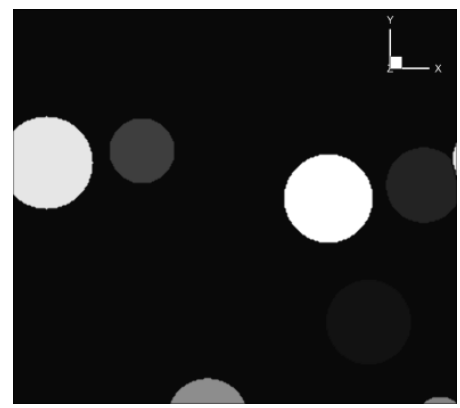

(b)

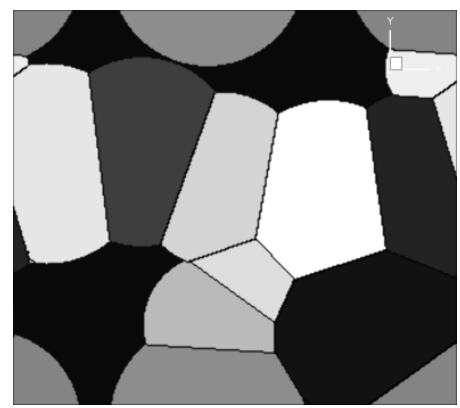

(e)

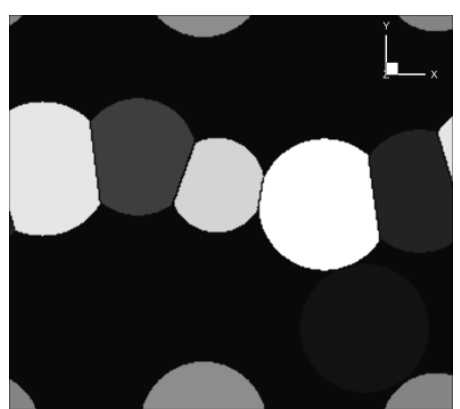

(c)

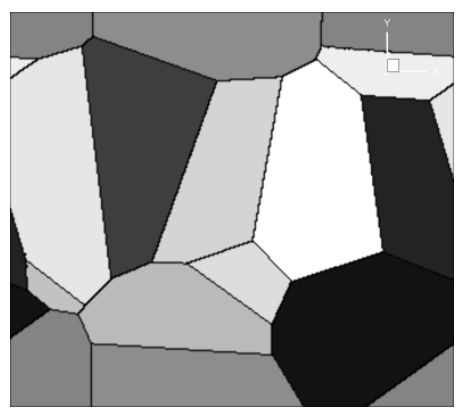

(f)

Figura 2 - Representação da microestrutura em 2D, simulada de forma aleatória nas interfaces da matriz com malhas de ortotetracaidecaedros, para $\mathrm{N}=28$ (a) nucleação, (b) $V_{v}=0,1$, (c) $V_{v}=0,3$, (d) $V_{V}=0,5$, (e) $V_{V}=0,7$, (f) 1,0 .

As microestruturas estudadas apresentam características geométricas aproximadamente perfeitas, pois a rede possui espaçamento cúbico de corpo centrado (CCC), com sítios de nucleação semelhantes e velocidade de crescimento constante. $\beta$ 
Desta forma, o que difere nos resultados das microestruturas representadas são as diferentes quantidades de núcleos. Na figura 3, é apresentado uma evolução microestrutural com 438 núcleos.

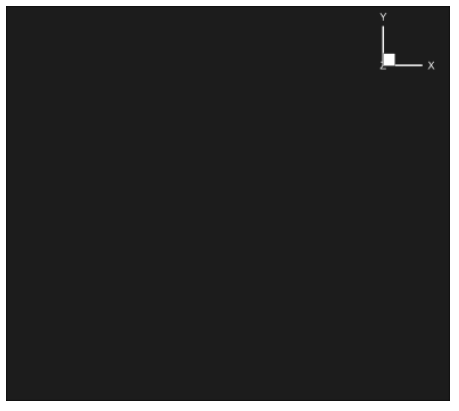

(a)

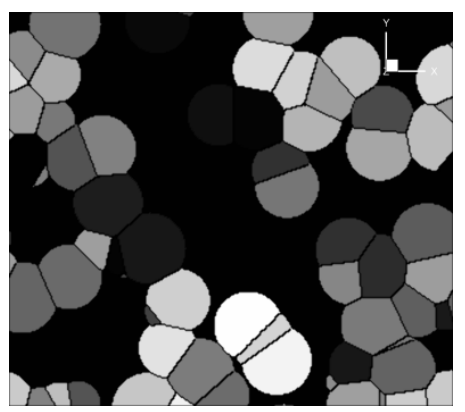

(d)

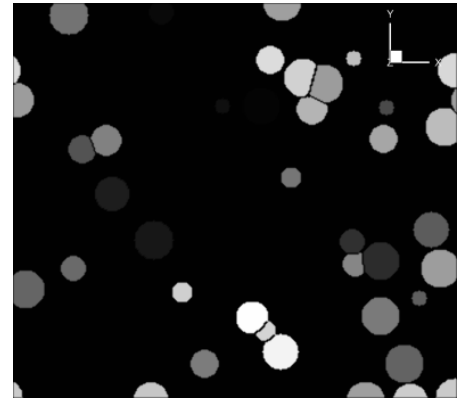

(b)

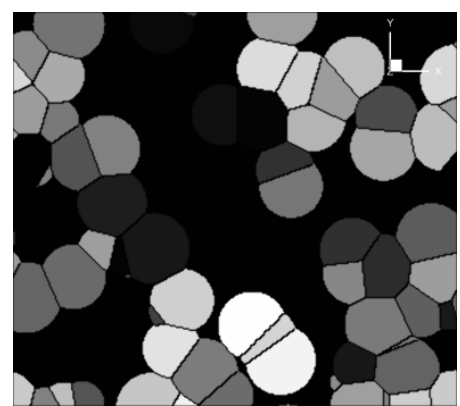

(e)

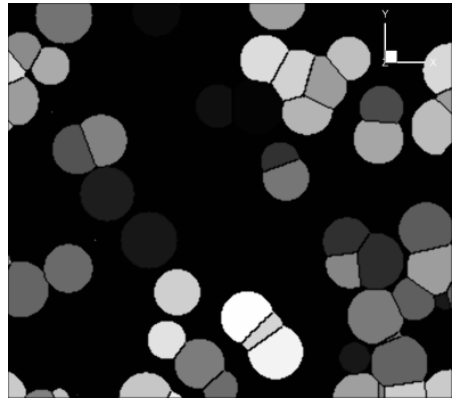

(c)

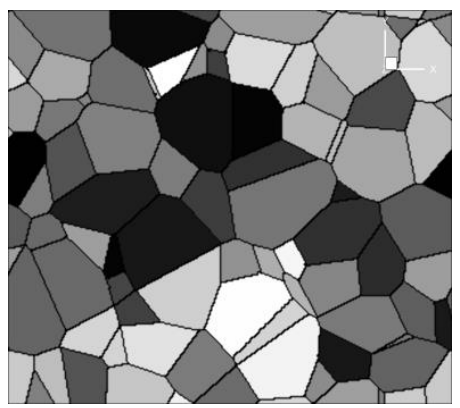

(f)

Figura 3 - Representação da microestrutura em 2D, simulada de forma aleatória nas interfaces da matriz com malhas de ortotetracaidecaedros, para $\mathrm{N}=438$ (a) nucleação, (b) $V_{V}=0,1$, (c) $V_{V}=0,3$, (d) $V_{V}=0,5$, (e) $V_{V}=0,7$, (f) 1,0 .

\section{1 - Fração Volumétrica}

$\mathrm{Na}$ figura 4, é apresentada a fração volumétrica $V_{V}$ em relação ao tempo de transformação para dois casos específicos: com 438 núcleos em 4a e 1040 em 4b.

No primeiro caso os resultados das simulações aleatórias, tanto nas interfaces, quanto na matriz, apresentam boa concordância com o modelo analítico de JMAK. Entretanto, a partir de mil núcleos, as simulações representando a nucleação nos contornos convergem para o modelo de Cahn, enquanto que a nucleação aleatória na matriz, continua com bom acordo em relação a JMAK. 


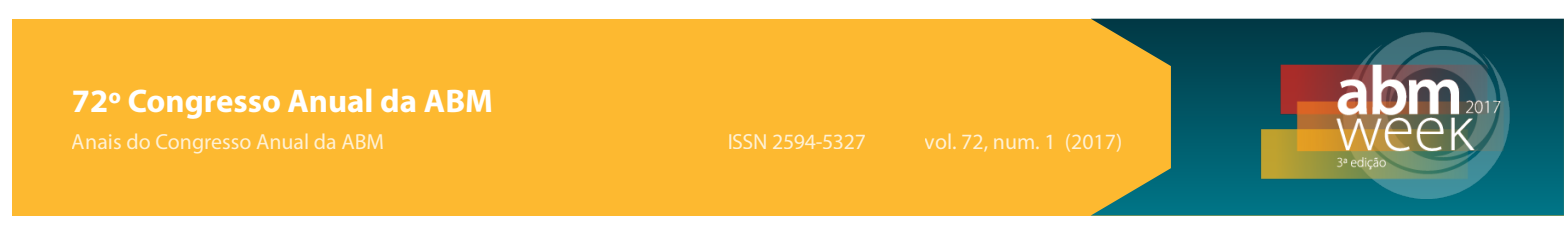

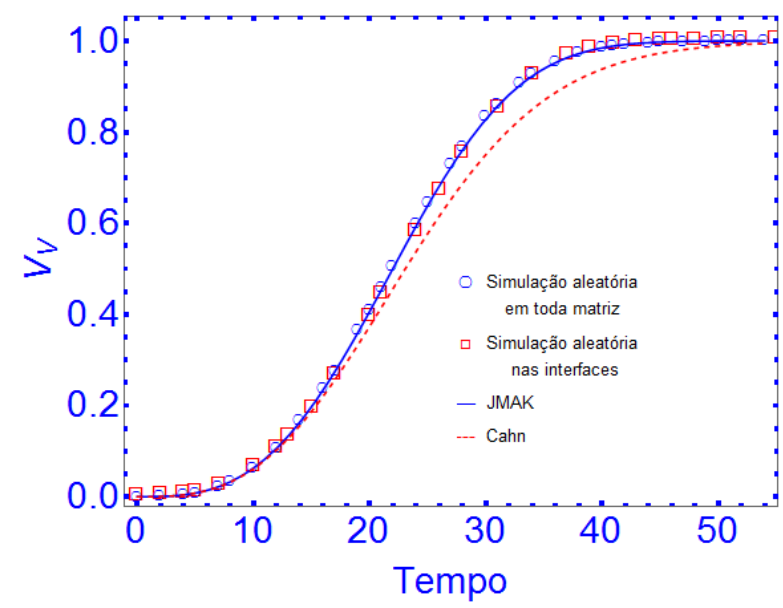

(a)

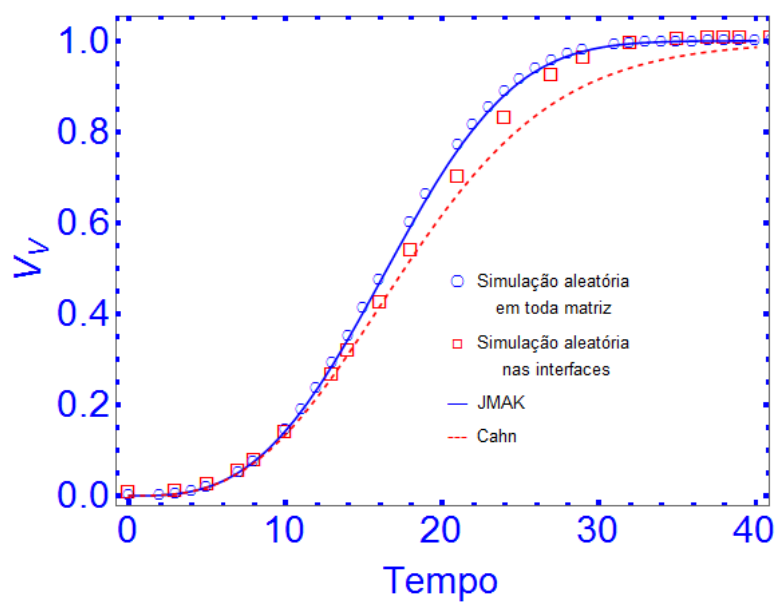

(b)

Figura 4 - Fração volumétrica $V_{v}$ em relação ao tempo, dos resultados obtidos a partir dos dados de simulações aleatórias nas interfaces de uma malha de ortotetracaidecaedro e aleatórias na matriz, (a) Tamanho de grão estimado em $40 \mu \mathrm{m}$, para $\mathrm{N}=438$, (b) Tamanho de grão estimado em $30 \mu \mathrm{m}$, para $\mathrm{N}=1040$.

\section{2 - Área Interfacial}

A figura 5 exibe o efeito do número de núcleos na evolução da densidade de área interfacial média, $S_{V}$, entre material transformado e não transformado. $S_{V}$ é importante pois permite visualizar com mais detalhes como a transformação ocorre, o que não é possível somente com a equação de JMAK.

$\mathrm{Na}$ figura $5 \mathrm{a}$, nota-se um bom acordo entre as simulações aleatórias na matriz e o modelo JMAK, porém o mesmo não ocorre entre as simulações aleatórias nas interfaces com o modelo de Cahn. Em $5 b$ a nucleação aleatória nas interfaces, apresenta em significativo desvio de JMAK, corroborando com o modelo de Cahn.

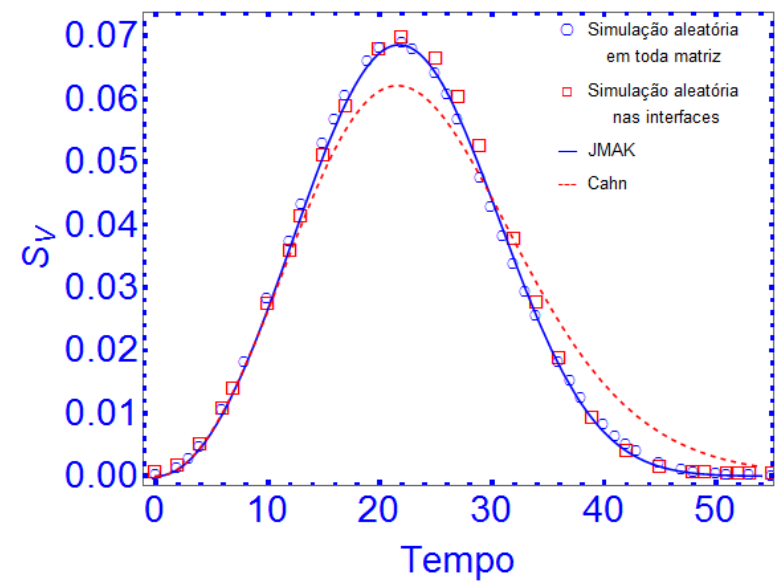

(a)

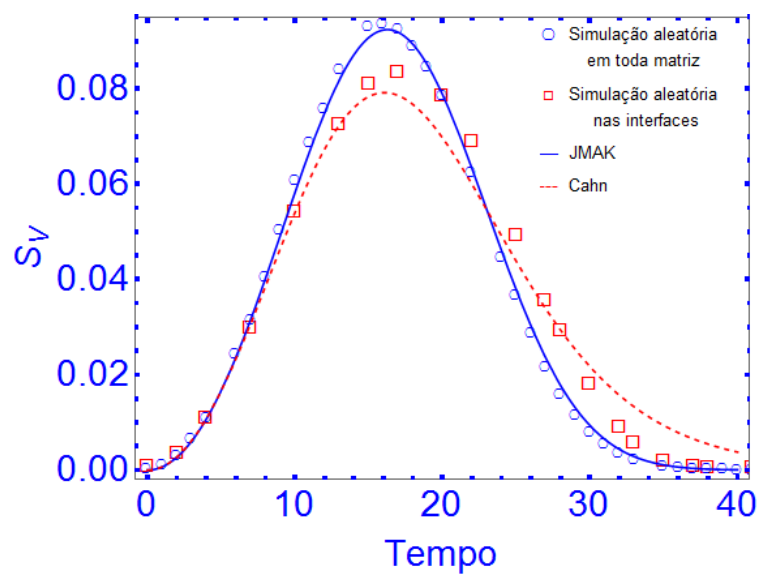

(b)

Figura 5 - Resultados da velocidade de avanço das interfaces versus Tempo, (a) Tamanho de grão estimado em $40 \mu \mathrm{m}$, para $\mathrm{N}=438$, (b) Tamanho de grão estimado em $30 \mu \mathrm{m}$, para $\mathrm{N}=1040$. 


\section{3 - Contiguidade}

Calculou-se as contiguidades, curva $\mathrm{C}_{\beta \beta}$ versus $\mathrm{V}_{\mathrm{v}}$, para os casos de simulações aleatórias na matriz e para as simulações nos contornos dos ortotetracaidecaedros.

Na figura 6 observar-se que a proporção da contiguidade varia de zero, em nenhuma transformação de nucleação, até um, representando uma transformação completa. Também é possível observar duas regiões bem definidas e distintas no gráfico.

A primeira define uma região que divide o gráfico ao meio, representado pelas simulações aleatórias na matriz e nas interfaces com até 438 núcleos. Nota-se que dos 8 aos 438, núcleos não ocorrem desvios significativos nos resultados. Este perfil se estende com os resultados com até 1000 núcleos, indicando que a nucleação ocorreu de forma aleatória.

Já as simulações com mais de mil núcleos, apresentam um desvio significativo na parte superior do gráfico, evidenciando, assim, a formação de clusters nos resultados das simulações aleatórias nas interfaces dos ortotetracaidecaedros.

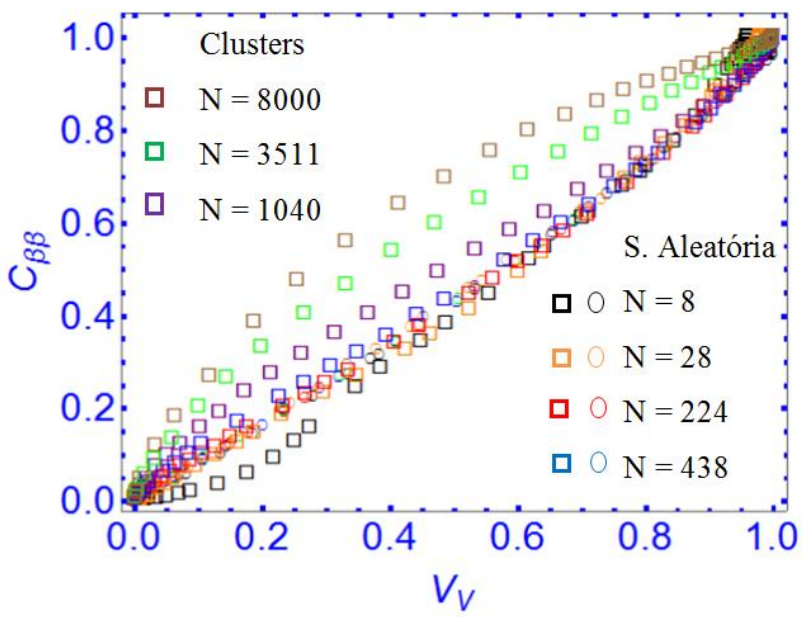

Figura 6 - Contiguidade versus $V_{v}$ para transformação aleatória na matriz e aleatória nas interfaces dos ortotetracaidecaedros.

\section{5 - Caminho Microestrutural}

A contiguidade é ratificada com os dados obtidos para o caminho microestrutural. É apresentado nas figura teta e tetinha uma relação entre a área interfacial entre as regiões transformadas e não transformadas, Sv, por unidade de volume e a fração volumétrica da região transformada $\mathrm{V} v$.

$\mathrm{Na}$ figura 7 os resultados corroboram com até mil núcleos corroboram os resultados anteriores, mostrando um bom acordo entre as simulações e JMAK. 


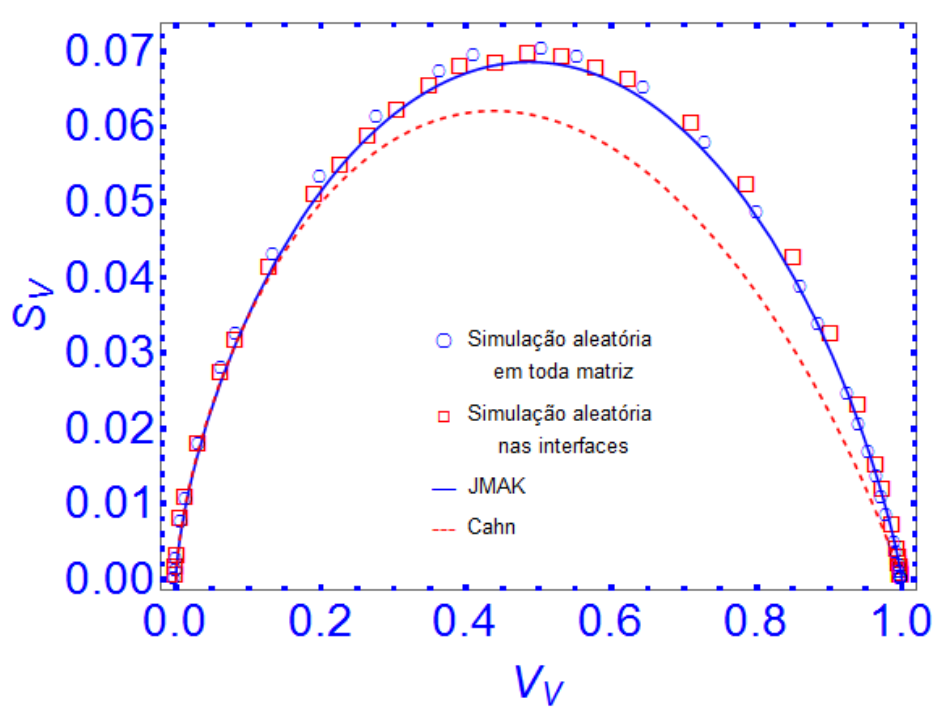

Figura 7. Resultados das simulações nas interfaces e na matriz, mostrando o caminho microestrutural, para $\mathrm{N}=438$.

A figura 8, mostra que o comportamento da curva com mais de mil núcleos apresenta uma acentuada projeção para a esquerda do centro da imagem. Observase também um bom acordo entre as simulações e o modelo de Cahn. Os resultados comprovam o comportamento da contiguidade em todos os casos.

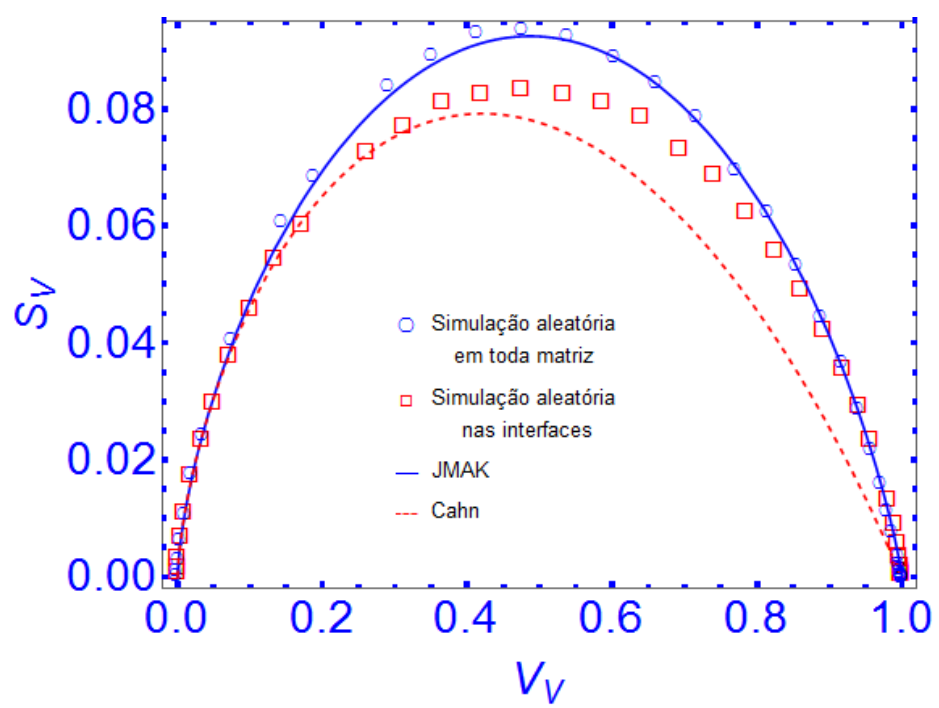

Figura 8. Resultados das simulações nas interfaces e na matriz, mostrando o caminho microestrutural, para $\mathrm{N}=1040$.

\section{CONCLUSÃO}

Aplicou-se com sucesso algumas técnicas de caracterização microestrutural nas microestruturas em 3D obtidas por simulação.

Os resultados mostram que há uma transição das curvas geradas, para os resultados com até mil núcleos, as curvas convergem com o modelo de JMAK. A partir deste valor os núcleos aproximam-se uns dos outros, formando-se clusters, aproximando-se do modelo analítico de Cahn para a nucleação em planos.

O estudo da contiguidade, comprovou a aleatoriedade dos fênomenos de nucleação e crescimento, simuladas com até mil núcleos e mostrou a presença de 
cluster a partir de mil núcleos. Os resultados foram ratificados com o caminho microestrutural, mostrando que o modelo adotado é uma boa opção para o estudo das transformações de fases.

\section{Agradecimentos}

Os autores agradecem a Deus, à Universidade Federal Fluminense (UFF), CNPq, CAPES e FAPERJ.

\section{REFERÊNCIAS}

1 Johnson WA, Mehl RF. Reaction Kinetics in Processes of Nucleation and Growth, Trans. Metall. Soc. A.I.M.E. 135 (1939) 416-441.

2 Kolmogrov AN. On the Statistical Theory of Metal Crystallization. Isvetiia Acad. Nauk. SSSR-Seriia Mat. 1 (1937) 335-359.

3 Avrami M. Kinetics of Phase Change. I General Theory. J. Chem. Phys. 7 (1939) 1103. doi:10.1063/1.1750380.

4 Avrami M. Kinetics of Phase Change II. Transformation - Time Relations for Random Distribution of Nuclei. J. Chem. Phys. 8 (1940) 212-224.

5 Avrami M. Granulation, Phase Change, and Microstructure Kinetics of Phase Change. III. J. Chem. Phys. 9 (1941) 177. doi:10.1063/1.1750872.

6 Rios PR, Villa E. Transformation kinetics for inhomogeneous nucleation. Acta Mater. 57 (2009) 1199-1208. doi:10.1016/j.actamat.2008.11.003.

7 Cahn JW. The kinetics of grain boundary nucleated reactions. Acta Metall. 4 (1956) 449-459.

8 Rios PR, Villa E, Assis WLS, Ribeiro TCS. Kinetics of transformations nucleated on random parallel planes: analytical modelling and computer simulation. Modelling Simulation Mater. Sci. Eng. 20, 2012.

9 Gurland J. The Measurement of Grain Contiguity in Two-Phase Alloys. Trans. Metall. Soc. AIME. 212 (1958) 452-455

10 Rios PR. Impingement function for nucleation on non-random sites. Acta Materialia, v. 55, n. 13, p. 4339-4348, 2007.

11 Vandermeer RA. Microstructural descriptors and the effects of nuclei clustering on recrystallization path kinetics. Acta Materialia, v. 53, n. 5, p. 1449-1457, 2005.

12 Vandermeer RA, Masumura RA. Microstructural path of grain-boundary-nucleated phase transformations. Acta metallurgica et materialia, v. 40, n. 4, p. 877-886, 1992.

13 Vandermeer RA, Jensen DJ. Microstructural path and temperature dependence of recrystallization in commercial aluminium. Acta Materialia, v. 39, p. 2083-2094, 2001. 